\title{
Does higher severity really correlate with a worse quality of life in obsessive-compulsive disorder? A meta-regression
}

This article was published in the following Dove Press journal: Neuropsychiatric Disease and Treatment

\author{
Andrea Pozza' \\ Christine Lochner ${ }^{2}$ \\ Fabio Ferretti ${ }^{\prime}$ \\ Alessandro Cuomo ${ }^{3}$ \\ Anna Coluccia' \\ 'Department of Medical Sciences, \\ Surgery and Neurosciences, Santa \\ Maria alle Scotte University Hospital \\ of Siena, Siena, Italy; ${ }^{2} \mathrm{SU} / \mathrm{UCT}$ \\ MRC Unit on Anxiety and Stress \\ Disorders, Department of Psychiatry, \\ Stellenbosch University, Cape \\ Town, South Africa; ${ }^{3}$ Department \\ of Molecular Medicine, University \\ of Siena School of Medicine and \\ Department of Mental Health, \\ University of Siena Medical Center \\ (AOUS), Siena, Italy
}

Background: Obsessive-compulsive disorder (OCD) is one of the leading causes of disability and reduced quality of life (QOL), with impairment in a number of domains. However, there is a paucity of literature on the association between severity of OCD symptoms and QOL, and the data that do exist are inconsistent. In addition, the role of severity in QOL has not been summarized as yet from a cross-generational perspective (ie, across childhood/adolescence and adulthood). Through meta-regression techniques, the current study summarized evidence about the moderator role of severity of OCD symptoms on differences in global QOL between individuals with OCD and controls.

Methods: Online databases were searched, and cross-sectional case-control studies comparing participants of all ages with OCD with controls on self-report QOL measures were included. Random-effect meta-regression techniques were used to comment on the role of illness severity in global QOL in individuals with OCD.

Results: Thirteen studies were included. A positive significant association emerged between OCD severity and effect sizes on global QOL: in samples with higher severity, there were narrower differences in QOL between patients with OCD and controls than in samples with lower severity. Such positive association was confirmed by a sensitivity analysis conducted on studies including only adults, where the difference in QOL ratings between patients and controls was significantly narrower when OCD severity was higher. Conversely, a negative association between severity and QOL was found in those studies including only children/adolescents, where the difference in QOL was significantly larger between patients and controls when OCD severity was higher.

Conclusion: QOL remains an important issue to address in the management of OCD in all age groups, irrespective of illness severity. Even in those with lower severity ratings, QOL may be considered as an important marker of treatment response.

Keywords: obsessive-compulsive disorder, quality of life, systematic review, symptoms severity, well-being, meta-regression

\section{Introduction}

Obsessive-compulsive disorder (OCD) - characterized by intrusive, recurrent thoughts, impulses, or mental images as well as repetitive overt or covert behaviors - is one of the leading sources of disability globally. ${ }^{1}$ The definition of health by the World Health Organization ${ }^{2}$ emphasizes the importance of subjective experience besides absence of disease as an essential component of quality of life (QOL). QOL can be conceptualized as "a patient's perceptions of the impact of disease in a variety of dimensions, including physical, mental, and social domains". ${ }^{3}$ Besides being one of the
Correspondence: Fabio Ferretti

Department of Medical Sciences, Surgery and Neurosciences, Santa Maria alle

Scotte University Hospital of Siena, Viale

Bracci 16, 53100 Siena, Italy

Tel +39577586408

Email ferrefa@unisi.it
Neuropsychiatric Disease and Treatment 2018:14 1013-1023

(c) (1) (8) ( 2018 Pozza et al. This work is published and licensed by Dove Medical Press Limited. The full terms of this license are available at https:/www.dovepress.com/terms.php cc. hereby accept the Terms. Non-commercial uses of the work are permitted without any further permission from Dove Medical Press Limited, provided the work is properly attributed. For permision for commercial use of this work, please see paragraphs 4.2 and 5 of our Terms (https://www.doveperess. com/terms.php). 
measures of the efficacy of existing treatments, the investigation of QOL appears to be a relevant - yet, not sufficiently recognized - topic in OCD research and practice. ${ }^{4,5}$ There is abundant evidence to suggest that QOL is significantly reduced in OCD compared to healthy controls. ${ }^{6,7}$ Even at the end of a successful treatment, individuals with OCD can still experience poorer QOL than normative samples. ${ }^{8}$ The only patient group with lower perceived QOL than individuals with OCD was those with schizophrenia. ${ }^{9}$

Some heterogeneity was found in the results about the relation between OCD and QOL, ${ }^{6,7}$ and a moderator of these findings could be different levels of OCD severity of the participants included in different studies. Clinical observation would suggest that individuals with higher severity experience poorer QOL. In the literature, however, few and inconsistent data is available on the association between symptom severity and QOL. For example, greater severity was related to poorer QOL in some studies, ${ }^{10}$ but this was not confirmed by others. ${ }^{11}$ In addition, information on the role of illness severity in QOL of OCD across different age groups (ie, adult vs children/adolescents) does not exist. The currently available research results have not yielded conclusive evidence about the association between illness severity and QOL. Thus, this inconsistency could concern differences related to the age cohorts, because the intensity and the direction of the association between QOL and severity could be different across adults and children/ adolescents with OCD. On one hand, in adult samples, some studies indicated a strong significant inverse association between QOL and severity, ${ }^{10}$ whereas other reported only a modest association; ${ }^{12}$ in others, a significant association was not found, ${ }^{11}$ and in some other studies only obsessions severity, but not compulsions, was associated with QOL. ${ }^{13}$ On the other hand, in children and adolescent samples, recent research more consistently demonstrated inverse relations between QOL and OCD symptom severity. ${ }^{14-16}$ It could be hypothesized that young people (children and adolescents) and adult groups with OCD have different clinical characteristics and levels of severity, as suggested by previous research demonstrating that, compared with adults, children and adolescents had considerably more severe aggressive obsessions, a higher proportion of males suffering from OCD, and lower insight of symptoms, whereas adults had stronger sexual obsessions. ${ }^{17}$ Thus, the generational cohort could have an impact on QOL, as the developmental phase can be associated with different levels of severity.

Using meta-regression techniques, the present study summarized evidence about the moderating role of OCD severity on the differences in global QOL between individuals diagnosed with OCD and controls. Given the differences in terms of severity between these groups, the role of illness severity in QOL was investigated separately for adults and children/ adolescent individuals with OCD. Knowing the role of severity in QOL can inform practice and facilitate treatment by directing the focus of clinicians to assessing and improving QOL as a function of severity presented by the patient.

\section{Methods}

\section{Eligibility criteria}

According to the PRISMA guidelines ${ }^{18}$ for observational cross-sectional case-control studies, the criteria for inclusion of studies involved the following domains.

\section{Participants}

Studies were included if conducted on participants who met diagnostic criteria for primary OCD. Diagnosis had to be made through a semi-structured interview based on standardized diagnostic criteria, such as the Structured Clinical Interview for DSM-IV Axis I disorders (SCID-I). ${ }^{19}$ Studies including participants with a lifetime or subclinical diagnosis of OCD were excluded as only current full diagnoses were considered. Studies were included if they used outpatients or inpatients and if they assessed patients with a diagnosis of mild to severe OCD. Participants of all ages were included. Studies on primary hoarding were excluded, as it is classified as a separate diagnosis in Diagnostic and Statistical Manual of Mental Disorders-fifth edition (DSM-5), ${ }^{1}$ not necessarily correlated with OCD, despite being included in the chapter on OCD-related disorders. Any psychiatric comorbidity and concurrent treatment - both psychological and pharmacological were not considered as reasons for exclusion. The rationale for this was the necessity to allow generalizability of findings to real-world practice, as a relevant proportion of patients with OCD presents with comorbidities or is under treatment. ${ }^{20}$

\section{Outcomes}

Studies were included if they used self-report validated questionnaires as measures of global QOL, such as The Medical Outcome Study 36-short-form health survey (SF-36). ${ }^{21}$ Measures had to assess QOL as a global construct. For those measures including subscales related to different domains of QOL, a global QOL measure was obtained by pooling scores on all the subscales and calculating a mean score as a global score. In addition, studies had to report data on OCD severity, measured by the Yale Brown Obsessive Compulsive Scale (Y-BOCS) ${ }^{22}$ for adults, or the Children-Yale Brown 
Obsessive Compulsive Scale (CY-BOCS $)^{23}$ for children and adolescents. The Y-BOCS is the gold standard measure to assess severity of OCD in adults - the most widely used measure in clinical assessment and trials. This scale consists of 10 items covering the length of time occupied by the symptoms, the associated distress, level of impairment, extent of resistance, and the extent of control over obsessions and compulsions. Each item is scored on a Likert-type scale ranging from 0 to 4 ; total scores of the scale range from 0 to 40. Similarly, the CY-BOCS is the gold standard measure of severity in children/adolescents, assessed over the preceding week with scores from 0 to 40 , with higher scores indicating increased levels of severity. It is commonly used as a primary screening and outcome measure in trials for youth with OCD. ${ }^{23}$

\section{Control groups}

Studies were included if the comparator groups included screened control participants, who have not reported any psychiatric disorder during a clinical interview, or unscreened individuals from the general population.

\section{Design}

Studies were included if they were based on an observational cross-sectional case-control design, where groups of participants with primary OCD diagnosis were compared with control groups on global QOL.

\section{Information sources and search procedure}

An online search of literature (published between 1966 and August 2017) was carried out. Keywords related to OCD ("Obsessive-compulsive disorder", "obsessions", "compulsions", "rituals") were combined through the Boolean operator "AND" with keywords related to QOL ("Quality of Life", "psychological well-being", "satisfaction with life", "global functioning", "social functioning", "family functioning", "subjective well-being") and with keywords related to QOL measures ("Medical Outcome Survey Short Form-36", "Quality of Life Inventory", "Satisfaction with Life Scale", "WHOQOL-100", "WHOQOL-BREF"). The following databases were searched: PubMed, Science Direct, and PsycINFO. Two reviewers (AP and FF) independently conducted the systematic search.

\section{Study selection and coding}

Studies were assessed on eligibility criteria by two independent reviewers (AP and FF) during three different stages.
When assessing the studies, each reviewer was blind to the assessment outcomes of the other reviewer. The contribution of each of the reviewers was the assessment of all the studies obtained by the search strategy and the screening stages at title, at abstract, and at full-text. Moreover, they independently coded the included studies, extracted the data, and inserted them in a worksheet; finally, each of the two reviewers independently calculated the summary measures.

During the first and the second stages, the eligibility of studies was evaluated after reading the title and the abstract, respectively. During these stages, studies were retained when there was no agreement on inclusion between the reviewers. Finally, the remaining studies were assessed on eligibility by reading the full-text article. After each stage of the selection, the reviewers discussed reasons for inclusion, and potential discrepancies in the judgment were addressed during meetings with another independent reviewer to obtain a shared pool of included studies for the meta-regression study. ${ }^{12,15,27-37}$

The independent reviewers (AP and FF) coded OCD severity as a moderator variable, extracted the data from the primary studies, and entered them into an Excel worksheet. Subsequently, during meetings with the third reviewer (AC), insertion of the data in the worksheets was checked for accuracy, and each potential discrepancy was discussed and resolved.

OCD severity was coded independently by the reviewers (AP and FF) by extracting the mean score of OCD and control groups on Y-BOCS or CY-BOCS. QOL was coded by extracting mean scores and standard deviations (SDs) on all the measures of QOL used by the study.

\section{Meta-analysis and meta-regression \\ Data extraction and summary measures}

Because a significant heterogeneity was expected in the effect sizes, standardized mean differences (SMDs) in effect sizes were calculated using random-effect meta-analyses. Random effects models assume that the included studies are drawn from populations of studies that systematically differ from each other. According to this model, the effect sizes extracted from the included studies differ not only because of the random error within studies (as in the fixed effects model), but also because of true variation in effect sizes from one study to the other. ${ }^{24}$ Data were extracted independently by two reviewers (AP and FF) through the following formula: ${ }^{25}$ $M_{O C D}-M_{C O N T R O L} / S D_{P O O L E D}$, where $M_{O C D}$ represents the mean of groups with OCD, $M_{\text {CONTROL }}$ is the mean of controls, and $S D_{\text {POOLED }}$ is the pooled SD. Then, the effect size of each 
study was weighted through the application of the following correction formula: $W_{\mathrm{zr}}=1 / S E_{\mathrm{zr}}^{2}$, where $S E_{\mathrm{zr}}^{2}$ is the standard error (SE) of the effect size computed for each study. This formula was corrected through the following formula for sampling error bias: $W^{\prime}=W_{\mathrm{zr}}\left(\alpha_{\mathrm{QOL}}\right) *\left(\alpha_{\mathrm{OCD}}\right)$, where $\alpha_{\mathrm{QOL}}$ is the Cronbach's reliability coefficient for QOL measures and $\alpha_{O C D}$ is the coefficient for OCD measures. Effect sizes were estimated using a 99\% confidence interval (CI) and interpreted according to criteria proposed by Cohen: ${ }^{25}$ values equal to 0.80 or higher were interpreted as large, up to 0.50 as moderate, and up to 0.20 as small. Hedges' correction for small sample bias was applied to all effect sizes. ${ }^{26}$ Negative effect sizes indicated larger differences between individuals with OCD and controls, with higher QOL among controls than participants with OCD.

A global effect size was calculated as an SMD in effect sizes by combining effect sizes related to total scores in different measures of QOL as a global construct. When a study reported data on different domains of QOL, such as work, social, or family QOL, a mean effect size was calculated by pooling mean scores on all domains. Subsequently, sensitivity analyses were conducted for calculating the SMDs effect sizes separately for studies including only adults, for those including only adolescents/children, and for studies including only screened healthy controls without psychiatric diagnoses. The rationale for calculating standardized estimates separately for adults and children/adolescents was the empirical evidence that children and adolescents report similar clinical features, whereas adults and children/ adolescents have differences in terms of the severity and clinical picture of OCD. ${ }^{17}$ Meta-regression analyses were conducted by calculating weighted least squares metaregressions, where dependent variables were SMDs in effect sizes of QOL between groups with OCD and controls, and independent variables were mean scores on the Y-BOCS or the CY-BOCS. In the meta-regression, positive values of Beta $(\beta)$ indicated that higher severity was associated with higher SMDs in effect sizes favoring controls, suggesting that in samples with higher severity there were higher SMDs in effect sizes between individuals with OCD and controls favoring the latter group.

To investigate heterogeneity in the SMDs in effect sizes, the $I^{2}$ and the $Q$ index were used. The $I^{2}$ index represents a measure of variation in percentage across studies that is attributable to heterogeneity rather than chance. A value approximating to zero suggests homogeneity, whereas values of $25 \%-50 \%, 50 \%-75 \%$, and $75 \%-100 \%$ represent low, medium, and large heterogeneities, respectively. ${ }^{24}$ The $Q$ statistic is computed by summing the squared deviations of each study's effect estimate from the overall effect estimate, while weighting the contribution of each study by its inverse variance. Under the hypothesis of homogeneity among the effect sizes, the $Q$ statistic follows a chi-square distribution with $k-1$ degrees of freedom, $k$ being the number of studies. To investigate the likelihood that effect sizes were subjected to publication bias, the Duval and Tweedie's trim and fill procedure and the visual inspection of funnel plot were used. The funnel plot represents a scatter plot in which the SMDs in effect sizes calculated from the primary studies on the horizontal axis are plotted against an indicator of study precision, the SE, on the vertical axis. ${ }^{24}$ In the absence of bias, the graph resembles a symmetrical inverted funnel, because the SMDs in effect sizes derived from smaller studies scatter more widely at the bottom of the graph, with the spread narrowing with increasing precision among larger studies. If there is publication bias because smaller studies that report no significant SMDs in effect sizes remain unpublished, then the funnel plot appears asymmetrical. ${ }^{24}$ The meta-analysis was run by Comprehensive Meta-analysis software, version 2.00 .

\section{Results}

\section{Process of selection of the studies}

The electronic search produced 1,067 records after duplicates were removed. Of those studies, 1,015 were excluded on the basis of their titles or abstract content, as they focused on irrelevant constructs. Thus, 52 were screened at the full-text stage. Of those, 39 were excluded in consideration of the eligibility criteria. Specifically, nine were excluded for various reasons, including not reporting sufficient data to calculate effect sizes (eg, SDs) or to conduct the meta-regression (eg, scores on the Y-BOCS or the CY-BOCS were not reported), and not replying to invitations to provide the necessary data to the present authors. In addition, two studies were excluded as they did not include measures of QOL, and three studies were excluded as they did not use validated QOL measures. Twenty studies were excluded as they did not use a casecontrol design (ie, the study did not use a control group). Two studies were excluded as they used participants with a lifetime OCD diagnosis but without a concurrent OCD diagnosis. Two studies were excluded as one study included participants with subclinical OCD symptoms and the other included participants with a hoarding disorder. Another publication was excluded as it used the same data as those reported in a study already included in the meta-analysis. Finally, 13 studies remained and were included in the 
meta-regression ( $\mathrm{n}=1,785,282$ effect sizes overall) by consensus of the three independent reviewers. The flow chart describing the selection process is presented in Figure 1.

\section{Characteristics of the included studies}

All included studies were published in peer-reviewed journals. ${ }^{11,27-37}$ All studies included patients with current primary OCD, and a healthy control cohort. Ten studies included adults, ${ }^{27,30-36}$ one included children, ${ }^{28}$ one adolescents, ${ }^{37}$ and one focused its analysis on adults and adolescents. ${ }^{29}$ Participants in OCD groups were outpatients in all studies. Only two studies reported whether patients were on concurrent pharmacological treatments..$^{29,34}$ Only three studies reported the number of individuals with comorbid personality disorders (51\% in one study and $0 \%$ in two studies). The studies were globally representative, with four having been conducted in European countries, ${ }^{14,27,31,36}$ six in American countries, ${ }^{11,28,30,31,34,37}$ and three in Asia. ${ }^{29,33,35}$ The description of the characteristics of each study is given in Table 1 .

\section{Comparison on QOL between individuals with OCD and controls}

This analysis included 13 studies $(\mathrm{n}=17,852)$. Results showed a large negative SMD in effect sizes favoring individuals with $\mathrm{OCD}(\mathrm{SMD}=-0.87, \mathrm{SE}=0.20,95 \% \mathrm{CI}:-1.26$ to $0.48, p<0.0001$ ), showing that individuals with $\mathrm{OCD}$ had significantly lower global QOL than controls. A large heterogeneity in the effect sizes of the studies was found
$\left(I^{2}=95.29, Q_{(12)}=254.86, p<0.0001\right)$. A forest plot which indicates the effect sizes of each study and the mean effect size is presented in Figure 2. Evidence for publication bias was not found, as suggested by the non-significant finding of the Egger test (Intercept $=-0.54, \mathrm{SE}=2.97,95 \% \mathrm{CI}:-7.08$ to $6.00, t_{(1)}=0.18$, two-tailed $p=0.85$ ). A funnel plot of publication bias for this comparison is provided in Figure 3, depicting the SEs of each study as a function of their corresponding effect sizes, indicated as SMDs in effect sizes.

\section{Meta-regression of QOL as a function of OCD severity in the total OCD cohort}

Because large heterogeneity was found in the meta-analysis comparing QOL between individuals with OCD and controls, a meta-regression of QOL as a function of illness severity was run in the OCD cohort. A positive significant association emerged between OCD severity and SMDs in effect sizes on QOL ( $\beta=0.10, \mathrm{SE}=0.01,99 \% \mathrm{CI}: 0.09-0.11, k=82$, $\mathrm{n}=162,590, p<0.01$ ), indicating that higher scores on OCD severity were associated with positive SMDs in effect sizes: samples with higher OCD severity reported narrower differences in QOL among participants with OCD and controls. Narrower differences suggested lower differences in terms of QOL between the patient groups and the controls. Such a positive association was confirmed $(\beta=0.18, \mathrm{SE}=0.01$, $99 \%$ CI: $0.16-0.20, p<0.01$ ) when sensitivity analysis was run including only screened controls $(k=31, \mathrm{n}=3,733)$ and excluding controls recruited from the general population.

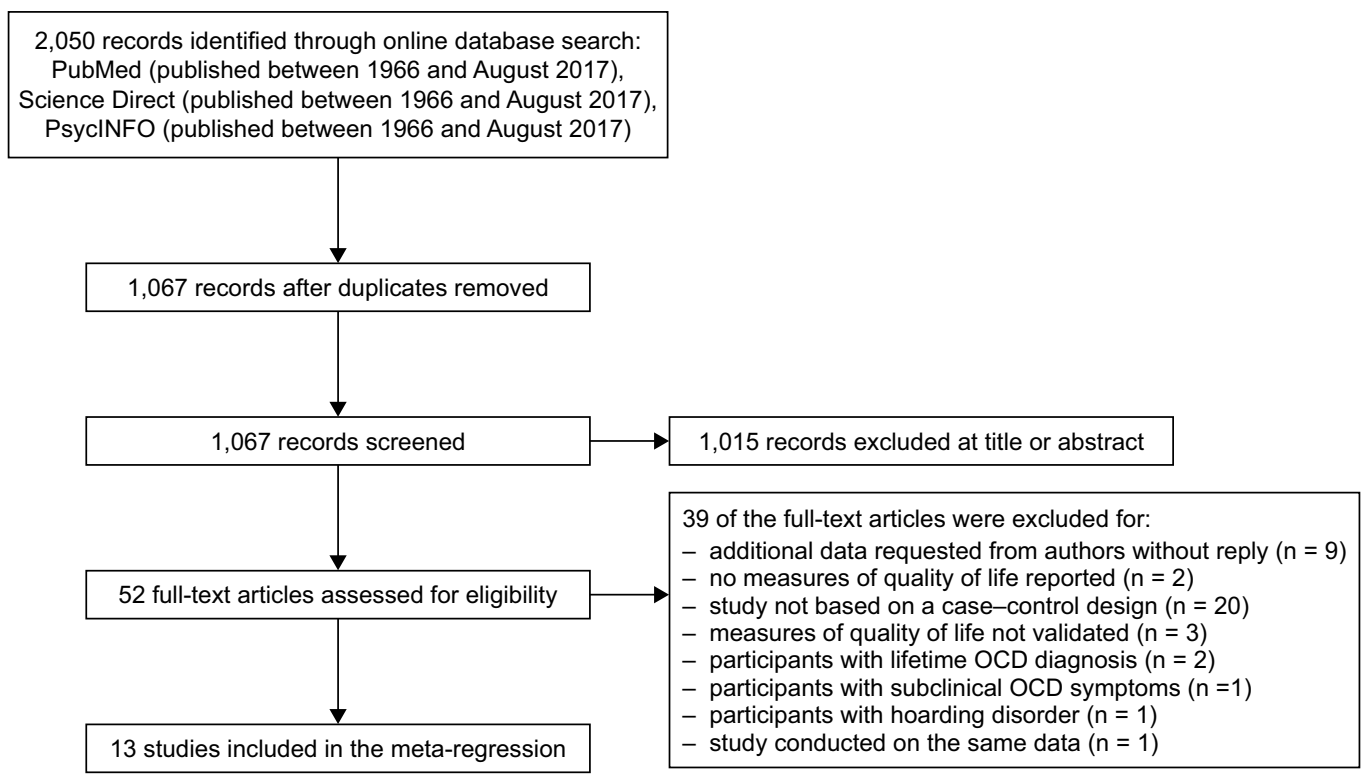

Figure I PRISMA flowchart of the study selection. Abbreviation: OCD, obsessive-compulsive disorder. 


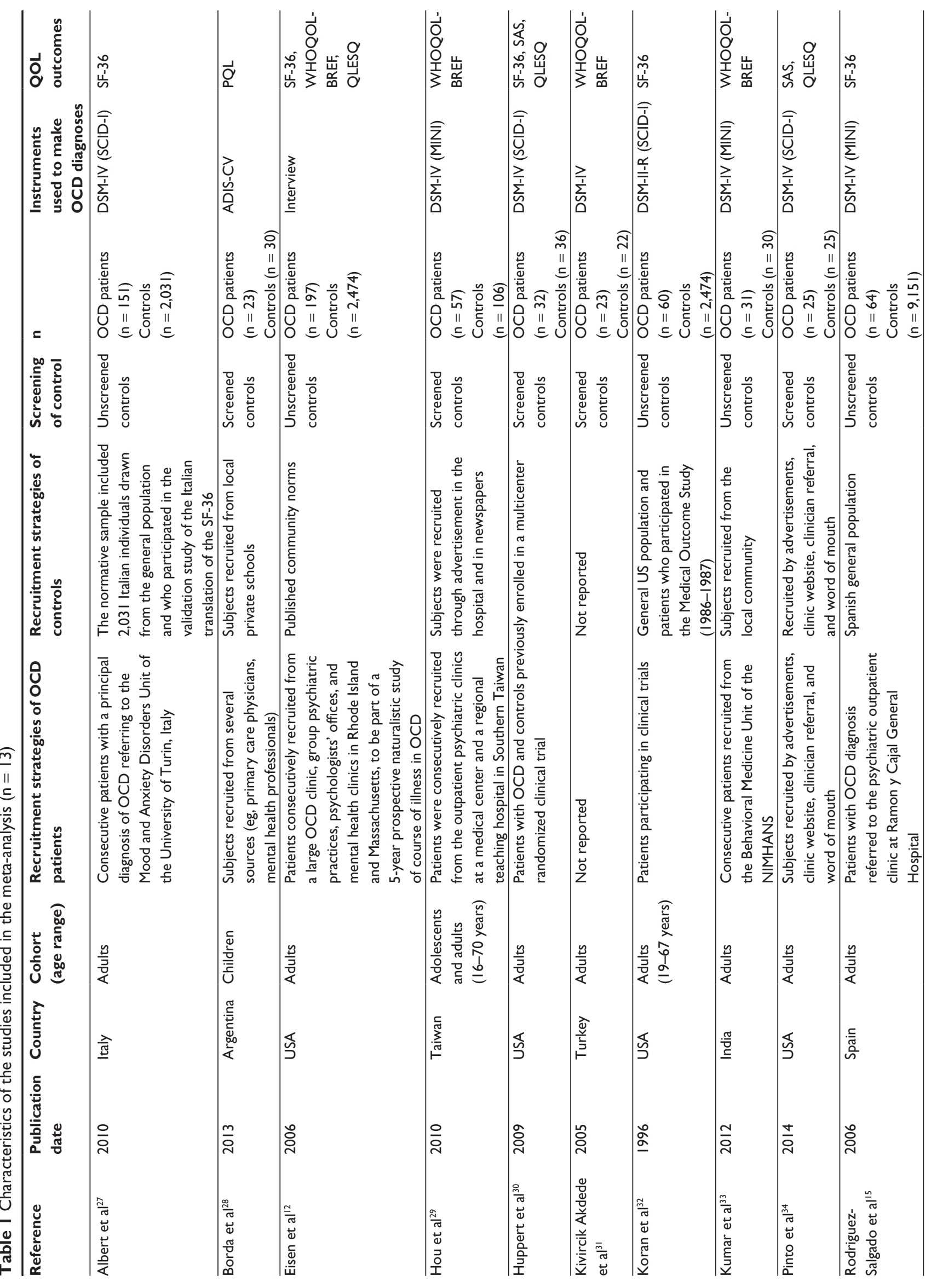




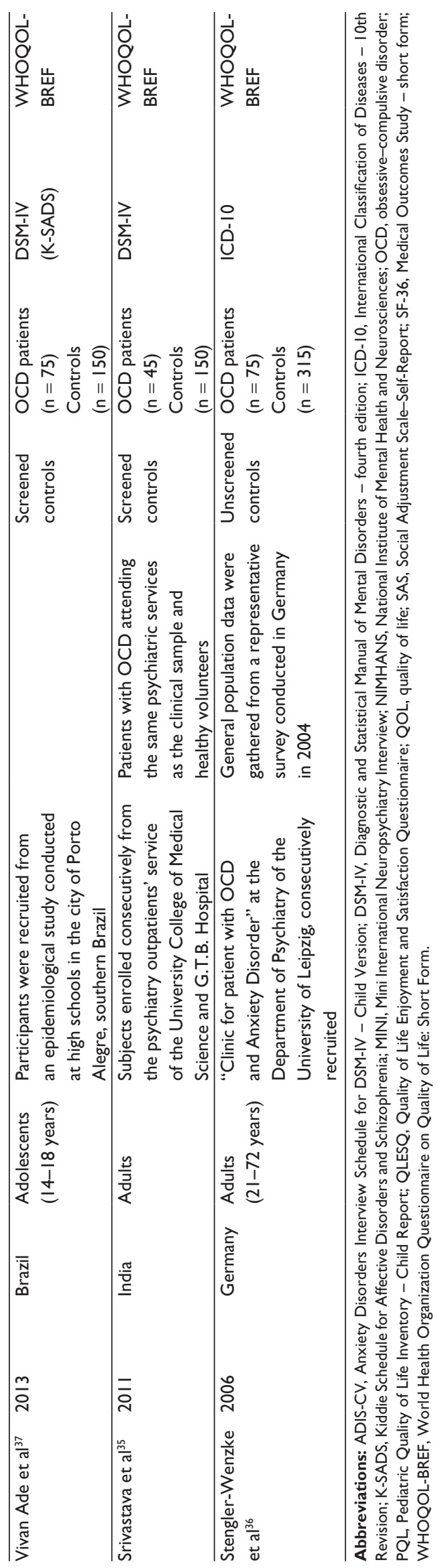

A meta-regression of global QOL as a function of OCD severity is presented in Figure 4.

\section{Meta-regression of QOL on severity in adults and children/adolescents separately}

When a sensitivity analysis was carried out, including only studies on adults, a positive association was still found $(\beta=0.10, \mathrm{SE}=0.01,99 \%$ CI: $0.09-0.11, p<0.01)$. When the analysis was restricted to children/adolescent samples, a negative association emerged $(k=6, \mathrm{n}=1,178)(\beta=-1.06$, $\mathrm{SE}=0.20,99 \% \mathrm{CI}:-1.46$ to $0.65, p<0.01)$, indicating that higher scores on severity were associated with wider, negative SMDs in effect sizes: samples with higher OCD severity reported wider differences on QOL among participants with OCD and controls favoring controls. Wider negative standardized differences in means favoring controls suggested that, for samples with higher severity, controls had higher mean scores than patients with OCD.

\section{Discussion}

In the current literature, findings on the association between illness severity of OCD symptoms and QOL are limited and inconsistent across studies, and a systematic review with meta-regression is not yet available. In addition, differences in the role of illness severity in QOL were not summarized across cohorts of adults and children/adolescents. Knowing these aspects can inform routine practice by focusing clinicians on assessing and improving QOL according to the severity presented by the patient. The current study used meta-regression techniques to provide a quantitative synthesis about the role of severity as a moderator of the differences in global QOL between individuals with OCD and controls. In addition, OCD severity was investigated separately for cohorts of adults and children/adolescents.

Thirteen case-control cross-sectional studies were included, where clinical groups with a diagnosis of $\mathrm{OCD}$ were compared with control groups from the general population. Severity of symptoms was assessed through the gold standard measures of OCD, the Y-BOCS, or the CY-BOCS. Results indicated a moderate negative effect size favoring control groups without evidence of publication bias: individuals with OCD had significantly lower QOL than controls. These findings were consistent with clinical impressions, suggesting that suffering from OCD can negatively impact the subjective experience of health. In addition, such results supported previous evidence that suggested a significant impairment in QOL among individuals with OCD confirming that this 


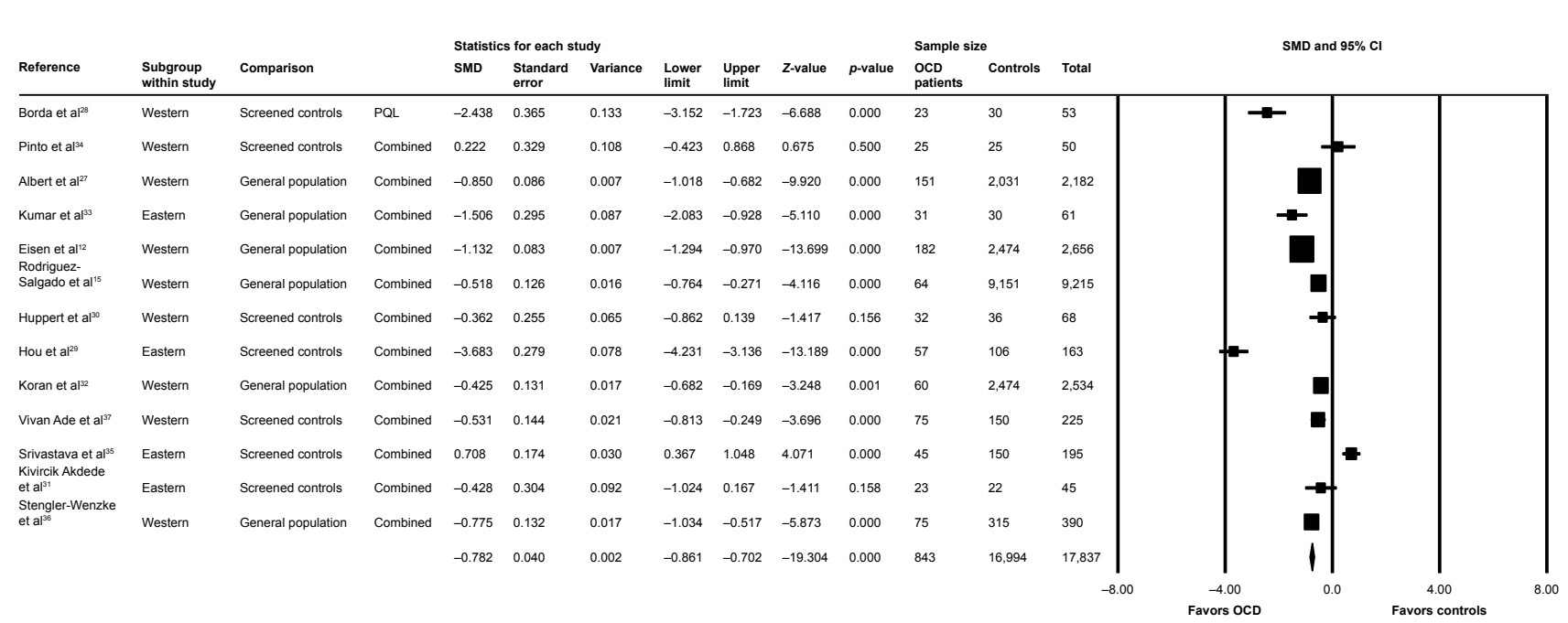

Figure 2 Forest plot between groups with OCD and controls on global QOL $(n=13)$.

Abbreviations: $\mathrm{Cl}$, confidence interval; OCD, obsessive-compulsive disorder; PQL, Pediatric Quality of Life Inventory - Child Report; QOL, quality of life; SMD, standardized mean difference.

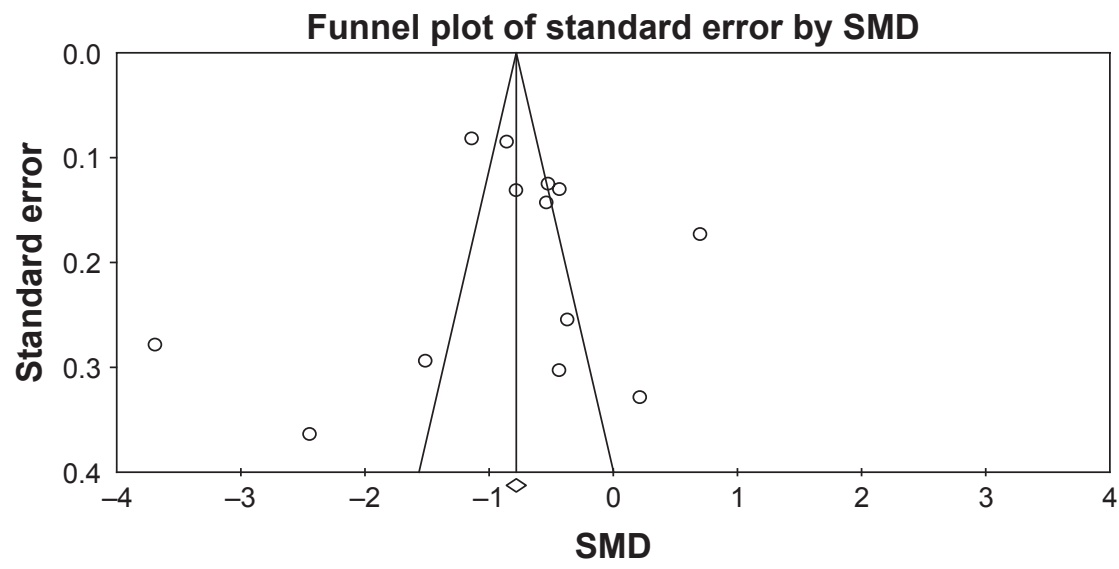

Figure 3 Funnel plot of publication bias for the meta-analysis between groups with OCD and controls on global QOL.

Abbreviations: OCD, obsessive-compulsive disorder; QOL, quality of life; SMD, standardized mean difference.

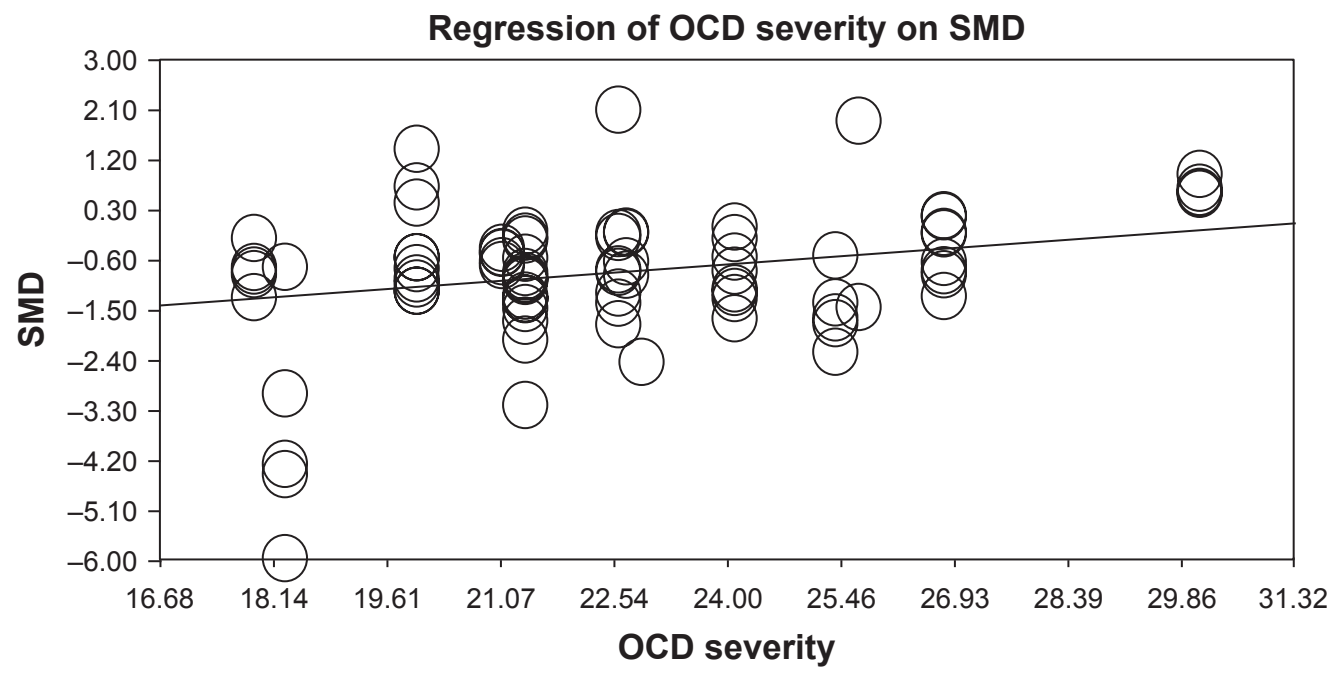

Figure 4 Meta-regression of global QOL as a function of OCD severity.

Abbreviations: OCD, obsessive-compulsive disorder; QOL, quality of life; SMD, standardized mean difference. 
condition is associated with a lack of subjective experience of health and well-being and not merely the presence of negative symptoms. ${ }^{6,7,30}$

Subsequently, because a large heterogeneity in the values of the SMDs in effects sizes was observed across the studies, a meta-regression was run with entering OCD severity as an independent variable and SMDs in effect sizes of global QOL as the outcome or dependent variable. Findings showed a significant positive association between the severity of OCD symptoms and SMDs in effect sizes in global QOL, indicating that, in studies with higher severity of OCD symptoms, the differences in global QOL between individuals with OCD and controls were less than the differences in global QOL between individuals with OCD with lower mean illness severity and controls. These findings were supported in a sensitivity analysis, where only studies with screened control groups were included and unscreened participants were removed. These findings appeared surprising and unexpected as clinical observations would suggest that individuals with more severe illness symptoms have poorer QOL than those with less severity. In addition, these results appeared in contrast with some previous studies in the literature reporting a negative relationship between OCD severity and QOL, ${ }^{10}$ where higher severity of OCD correlated with poorer QOL. However, this explanation cannot be conclusive as the current study did not control for OCD duration or age onset as indicators of chronicity. In addition, it could be expected that more severe individuals have lower life expectations than those with mild to moderate symptoms, and this would lead them to report higher perceptions of QOL. Moreover, it could be hypothesized that those individuals with more severe symptoms have a poorer insight of the disorder and its effects on QOL, because a poorer insight has been found to be a predictor of stronger treatment resistance and higher severity. ${ }^{38}$ Obsessions and compulsions, which are typically pervasive in more severe patients, can become a relevant part of their identity and personal values, and the most severe patients can view them as necessary and useful to maintain wellbeing. Thus, the attention of clinicians should be directed particularly to stimulate self-awareness of effects of OCD on QOL and to develop new values and life roles - different from those exclusively based on obsessions and compulsive behaviors. Again, due to the lack of studies investigating this aspect, insight was not accounted as potentially related to severity and QOL. A clinical implication could be the use of value-based psychotherapeutic components of the Acceptance and Commitment Therapy ${ }^{39}-\mathrm{a}$ form of therapy aimed to promoting the development of personal values as a therapeutic process. Another clinical implication of these findings could be the use of well-being therapy ${ }^{40}$ as a form of intervention particularly directed at improving perceptions of QOL. This form of psychotherapy - originally created for subclinical symptoms of depression - could be used for those individuals with less severe symptoms. Patients with less severe symptoms should be taken into account as a particularly vulnerable subgroup in terms of QOL, who should be provided with additional skills necessary to develop a better perception or appreciation of QOL. In contrast to the common belief that the severe patients are more vulnerable, thus needing more intensive treatments, these findings suggested that those with lower illness severity are also a vulnerable cohort with reduced QOL. It could be hypothesized that those individuals having less severe symptoms, who typically can continue working or having social relations despite OCD, would have strong awareness of the daily negative effects of OCD and experience a more intense sense of social stigma than those individuals with more severe OCD and those with weaker insight. In conclusion, comorbidity (eg, specific types of comorbidities or the number of comorbid disorders), stressors (eg, financial, educational, marital stressors, lack of support, etc), and the level of insight could be assessed in future research as moderators of the relationship between illness severity and OCD.

Subsequently, a sensitivity analysis was run, computing SMDs in effect sizes with studies on adults only. A further analysis was conducted with studies including only cohorts of adolescents/children. Although a positive association was found between severity of OCD symptoms and standardized differences of effect sizes, it became negative when the analysis was restricted to children and adolescent samples. In the latter samples, a negative association indicated that higher levels of OCD severity were associated with lower differences in mean effect sizes: samples where OCD severity was higher then reported lower global QOL among participants with OCD than controls. Evidence of a negative association between illness severity and QOL in children/adolescents makes clinical sense, but appears inconsistent with empirical and clinical observations that, in some youth - particularly children - there is generally poorer insight of OCD symptoms and lesser awareness of their consequences in daily life as compared with adults. ${ }^{1,17}$ It could be expected that a variable like family accommodation, particularly present in youth with more severe OCD, can moderate the relation between OCD severity and QOL; because, for children with higher 
severity, family accommodation is stronger and this can make the child/adolescent less aware of symptoms and their effects on QOL. ${ }^{40}$ Family accommodation can manifest in various forms including modifying family routines, engaging in the patient's compulsions, and facilitating avoidance of OCD triggers - thereby maintaining OCD symptomatology by disallowing the individuals to face their feared situations. ${ }^{41}$

\section{Limitations and conclusion}

Some limitations should be highlighted. As previously mentioned, other variables - potentially associated with QOL and severity, such as insight, symptoms duration, and family accommodation - were not considered, because this information was lacking in most of the studies we included. Further studies investigating the role of these variables are needed. Another limitation concerned the cross-sectional design of the studies: future longitudinal studies should investigate whether severity is prospectively associated with QOL. Moreover, the cross-sectional nature of the study prevented drawing firm conclusions about the causal relations between OCD and QOL. Another relevant shortcoming concerned the small number of studies including children and adolescents, which could be associated with a poor statistical power of the analysis; this highlights the need for further studies evaluating QOL in young people with OCD. Finally, specific domains of QOL were not investigated due to the small number of studies assessing them, such as QOL within the domains of social and family relationships.

In conclusion, the current study was the first one using meta-regression techniques to summarize evidence about the role of illness severity in global QOL in individuals diagnosed with OCD. In adults, the difference in QOL measures between patients and controls was significantly less when OCD severity was higher; conversely, in children/adolescents, the difference in QOL was significantly more between patients and controls when OCD severity was higher. QOL remains an important issue to address in the management of OCD in all age groups, irrespective of illness severity. Even in those with lower severity ratings, QOL may be considered an important marker of treatment response.

\section{Disclosure}

The authors report no conflicts of interest in this work.

\section{References}

1. American Psychiatric Association. Diagnostic and Statistical Manual of Mental Disorders (DSM-5). Washington, DC: American Psychiatric Association; 2013.
2. WHO. Constitution of the World Health Organization. Geneva: World Health Organization; 1948.

3. Varni JW, Burwinkle TM, Seid M, Skarr D. The PedsQL 4.0 as a pediatric population health measure: feasibility, reliability, and validity. Ambul Pediatr. 2003;3(6):329-341.

4. Lochner C, Mogotsi M, du Toit PL, Kaminer D, Niehaus DJ, Stein DJ. Quality of life in anxiety disorders: a comparison of obsessive-compulsive disorder, social anxiety disorder, and panic disorder. Psychopathology. 2003;36(5):255-262.

5. Moritz S. A review on quality of life and depression in obsessivecompulsive disorder. CNS Spectr. 2008;13(9 Suppl 14):16-22.

6. Coluccia A, Fagiolini A, Ferretti F, et al. Adult obsessive-compulsive disorder and quality of life outcomes: a systematic review and metaanalysis. Asian J Psychiatr. 2016;22:41-52.

7. Coluccia A, Ferretti F, Fagiolini A, Pozza A. Quality of life in children and adolescents with obsessive-compulsive disorder: a systematic review and meta-analysis. Neuropsychiatr Dis Treat. 2017;13:597-608.

8. Safren SA, Heimberg RG, Brown EJ, Holle C. Quality of life in patients with social phobia. Depress Anxiety. 1997;4:126-133.

9. Bobes J, González MP, Bascarán MT, Arango C, Sáiz PA, Bousoño M. Quality of life and disability in patients with obsessive-compulsive disorder. Eur Psychiatry. 2001;16(4):239-245.

10. Moritz S, Rufer M, Fricke S, et al. Quality of life in obsessive-compulsive disorder before and after treatment. Compr Psychiatry. 2005;46(6): 453-459.

11. Rapaport MH, Clary C, Fayyad R, Endicott J. Quality-of-life impairment in depressive and anxiety disorders. Am J Psychiatry. 2005;162(6): $1171-1178$.

12. Eisen JL, Mancebo MA, Pinto A, et al. Impact of obsessive-compulsive disorder on quality of life. Compr Psychiatry. 2006;47(4):270-275.

13. Masellis M, Rector NA, Richter MA. Quality of life in OCD: differential impact of obsessions, compulsions, and depression comorbidity. Can J Psychiatry. 2003;48(2):72-77.

14. Lack CW, Storch EA, Keeley ML, et al. Quality of life in children and adolescents with obsessive-compulsive disorder: base rates, parentchild agreement, and clinical correlates. Soc Psychiatry Psychiatr Epidemiol. 2009;44(11):935-942.

15. Rodriguez-Salgado B, Dolengevich-Segal H, Arrojo-Romero M, et al. Perceived quality of life in obsessive-compulsive disorder: related factors. BMC Psychiatry. 2006;6:20.

16. Storch EA, Small BJ, McGuire JF, Murphy TK, Wilhelm S, Geller DA. Quality of life in children and youth with obsessive-compulsive disorder. J Child Adolesc Psychopharmacol. Epub 2017 Sep 14.

17. Geller DA, Biederman J, Faraone S, et al. Developmental aspects of obsessive-compulsive disorder: findings in children, adolescents, and adults. J Nerv Ment Dis. 2001;189(7):471-477.

18. Shamseer L, Moher D, Clarke M, et al; PRISMA-P Group. Preferred reporting items for systematic review and meta-analysis protocols (PRISMA-P) 2015: elaboration and explanation. BMJ. 2015; 349: g7647.

19. First MB, Spitzer RL, Gibbon M, Williams JB. Structured Clinical Interview for DSM-IV Axis I Disorders-Patient Edition (SCID-I/P, version 2.0). New York: Biometrics Research Department; 1995.

20. Brown TA, Barlow DH. A proposal for a dimensional classification system based on the shared features of the DSM-IV anxiety and mood disorders: implications for assessment and treatment. Psychol Assess. 2009;21(3):256-271.

21. Ware JE Jr, Sherbourne CD. The MOS 36-item short-form health survey (SF-36). I. Conceptual framework and item selection. Med Care. 1992;30(6):473-483.

22. Goodman WK, Price LH, Rasmussen SA, et al. The Yale-Brown Obsessive Compulsive Scale: I. Development, use, and reliability. Arch Gen Psychiatry. 1989;46(11):1006-1011.

23. Scahill L, Riddle MA, McSwiggin-Hardin M, et al. Children's YaleBrown Obsessive Compulsive Scale: reliability and validity. J Am Acad Child Adolesc Psychiatry. 1997;36(6):844-852. 
24. Higgins JP, Thompson SG. Quantifying heterogeneity in a meta-analysis. Stat Med. 2002;21(11):1539-1558.

25. Cohen J. Statistical Power Analysis for the Behavioral Sciences. New York: Routledge; 1988.

26. Hedges L. Distribution theory for Glass' estimator of effect size and related estimators. J Educ Behav Stat. 1981;6(2):107-128.

27. Albert U, Maina G, Bogetto F, Chiarle A, Mataix-Cols D. Clinical predictors of health-related quality of life in obsessive-compulsive disorder. Compr Psychiatry. 2010;51(2):193-200.

28. Borda T, Feinstein BA, Neziroglu F, Veccia T, Perez-Rivera R. Are children with obsessive-compulsive disorder at risk for problematic peer relationships? J Obsessive Compuls Relat Disord. 2013;2(4): 359-365.

29. Hou SY, Yen CF, Huang MF, Wang PW, Yeh YC. Quality of life and its correlates in patients with obsessive-compulsive disorder. Kaohsiung J Med Sci. 2010;26(8):397-407.

30. Huppert JD, Simpson HB, Nissenson KJ, Liebowitz MR, Foa EB. Quality of life and functional impairment in obsessive-compulsive disorder: a comparison of patients with and without comorbidity, patients in remission, and healthy controls. Depress Anxiety. 2009;26(1): 39-45.

31. Kivircik Akdede BB, Alptekin K, Akvardar Y, Kitiş A. [Quality of life in patients with obsessive-compulsive disorder: relations with cognitive functions and clinical symptoms]. Turk Psikiyatri Derg. 2005; 16(1):13-19. Turkish [with English abstract].

32. Koran LM, Thienemann ML, Davenport R. Quality of life for patients with obsessive-compulsive disorder. Am J Psychiatry. 1996;153(6): 783-788.
33. Kumar A, Sharma MP, Kandavel T, Reddy YJ. Cognitive appraisals and quality of life in patients with obsessive-compulsive disorder. J Obsessive Compuls Relat Disord. 2012;1(4):301-305.

34. Pinto A, Steinglass JE, Greene AL, Weber EU, Simpson HB. Capacity to delay reward differentiates obsessive-compulsive disorder and obsessivecompulsive personality disorder. Biol Psychiatry. 2014;75(8):653-659.

35. Srivastava S, Bhatia MS, Thawani R, Jhanjee A. Quality of life in patients with obsessive-compulsive disorder: a longitudinal study from India. Asian J Psychiatr. 2011;4(3):178-182.

36. Stengler-Wenzke K, Kroll M, Matschinger H, Angermeyer MC. Quality of life of relatives of patients with obsessive-compulsive disorder. Compr Psychiatry. 2006;47(6):523-527.

37. Vivan Ade S, Rodrigues L, Wendt G, Bicca MG, Cordioli AV. Quality of life in adolescents with obsessive-compulsive disorder. Rev Bras Psiquiatr. 2013;35(4):369-374.

38. Twohig MP, Hayes SC, Plumb JC, et al. A randomized clinical trial of acceptance and commitment therapy versus progressive relaxation training for obsessive-compulsive disorder. J Consult Clin Psychol. 2010; 78(5):705-716.

39. Visser H, van Megen H, van Oppen P, et al. The impact of poor insight on the course of Obsessive-Compulsive Disorder in patients receiving naturalistic treatment. J Obsessive Compuls Relat Disord. 2017;13:42-48.

40. Fava GA, Rafanelli C, Cazzaro M, Conti S, Grandi S. Well-being therapy. A novel psychotherapeutic approach for residual symptoms of affective disorders. Psychol Med. 1998;28(2):475-480.

41. Wu MS, McGuire JF, Martino C, Phares V, Selles RR, Storch EA. A meta-analysis of family accommodation and OCD symptom severity. Clin Psychol Rev. 2016;45:34-44.
Neuropsychiatric Disease and Treatment

\section{Publish your work in this journal}

Neuropsychiatric Disease and Treatment is an international, peerreviewed journal of clinical therapeutics and pharmacology focusing on concise rapid reporting of clinical or pre-clinical studies on a range of neuropsychiatric and neurological disorders. This journal is indexed on PubMed Central, the 'PsycINFO' database and CAS,

\section{Dovepress}

and is the official journal of The International Neuropsychiatric Association (INA). The manuscript management system is completely online and includes a very quick and fair peer-review system, which is all easy to use. Visit http://www.dovepress.com/testimonials.php to read real quotes from published authors. 